\title{
Validation of Multiresidue Screening Methods for the Determination of 186 Pesticides in 11 Agricultural Products Using Gas Chromatography (GC)
}

\author{
Yoshichika Hirahara, ${ }^{*, a}$ Mika Kimura, ${ }^{a}$ Tomoko Inoue, ${ }^{a}$ Seiji Uchikawa, ${ }^{a}$ Shoji Otani, ${ }^{a}$ \\ Asami Haganuma, ${ }^{a}$ Nobuyuki Matsumoto, ${ }^{b}$ Asumi Hirata, ${ }^{b}$ Shiho Maruyama, ${ }^{b}$ \\ Tomomi lizuka, ${ }^{b}$ Masaho Ukyo, ${ }^{b}$ Mitsue Ota, ${ }^{b}$ Hideaki Hirose, ${ }^{a}$ Sosuke Suzuki, ${ }^{a}$ \\ and Yukinori Uchida ${ }^{a}$ \\ ${ }^{a}$ Kobe Quarantine Station, Center for Inspection of Imported Foods and Infectious Diseases, 1-1, Toyahama-cho, Hyogo-ku, Kobe \\ 652-0866, Japan and ' Yokohama Quarantine Station, Center for Inspection of Imported Foods and Infectious Diseases, 107-8, \\ Nagahama, Kanazawa-ku, Yokohama 236-0011, Japan
}

(Received July 14, 2005; Accepted July 28, 2005)

Validation of multiresidue screening methods for the determination of 186 pesticides in 11 agricultural products: broccoli, asparagus, carrot, spinach, burdock, matsutake mushroom, cauliflower, orange, soybean, sesame and millet was done by gas chromatography (GC). The investigated pesticides were selected on the based of such compounds that are commonly used around the world. Although the recovery of 58 of the pesticides was low $(<50 \%)$ in some crops, the 128 pesticides that spiked in samples at $0.1 \mathrm{mg} / \mathrm{kg}$ showed satisfactory recoveries $(\geq \mathbf{5 0 \%})$ in all crops with relative standard deviation of 4-21\%. These validated 128 pesticides were therefore newly acceptable for the pesticide-monitoring programme at the Quarantine Station in Japan; the quantitative limits ranged from 0.005 to $0.1 \mathrm{ppm}$ by GC on a crop basis. The screening methods were applied to monitor the residue from a total of 200 pesticides including 72 previously validated in imported foods at the Station in Japan. Pesticide residue from $188(12.4 \%)$ was found in 1516 samples. Of these, $4(0.26 \%)$ were in violation of Japanese maximum residue limits (MRLs). No detectable residue was found in $1328(87.6 \%)$ samples.

Key words — pesticide, validation, positive list system, screening method, imported food, gas chromatography

\footnotetext{
*To whom correspondence should be addressed: Kobe Quarantine Station, Center for Inspection of Imported Foods and Infectious Diseases, 1-1, Toyahama-cho, Hyogo-ku, Kobe 652 0866, Japan. Tel.: +81-78-672-9657; Fax: +81-78-672-9663; E-mail: y-hirahara@forth.go.jp
}

\section{INTRODUCTION}

Japan is going to implement a so-called "positive list system" a system to prohibit in principle the distribution of foods containing a certain level of agricultural chemicals in case where no maximum residue limits (MRLs) have been set. The agricultural chemicals include pesticides, veterinary drugs and feed additives. This activity is based on the amended Japanese Food Sanitation Law published in May 2003. The system will take effect no later than May 2006. At the time of its implementation, MRLs will exist for about 750 chemicals. Pesticides are applied to agricultural crops throughout the entire world to control undesirable insects, ${ }^{1,2)}$ mites, ${ }^{3,4}$ fungi, ${ }^{5,6)}$ weeds, ${ }^{7,8)}$ nematodes ${ }^{9,10)}$ and mollusks. ${ }^{11}$ Pesticide-monitoring programme in imported foods are therefore important to ensure that these pesticides do not exceed the MRLs. The monitoring programme at the Quarantine Station in Japan uses the multiresidue screening methods. Thus, the validation of the screening methods is administratively indispensable to judge the applicability of these pesticides by the monitoring programmes at the Station. In the previous paper, ${ }^{12)}$ we proposed a method by gas chromatography (GC) for rapid screening of 104 pesticides in vegetables, fruits, cereals and seeds for routine purposes. In this study, another 186 pesticides to be investigated were selected on the based of their common use around the world. The aim of this work was to validate these 186 pesticides by the proposed routine screening methods using eleven common agricultural products. Pesticide-monitoring programme was also assigned at the Quarantine Station in Japan and used as the method to monitor 
imported foods. The monitoring results are presented here.

\section{MATERIALS AND METHODS}

\section{Chemicals, Reagents and Materials}

Pesticide Standard: Certified reference pesticides were purchased from Hayashi Pure Chemical Industries (Osaka, Japan), Wako Pure Chemical Industries (Osaka, Japan) or Kanto Kagaku (Tokyo, Japan) (listed in Fig. 1). The purity of these pesticides was more than $97 \%$ by GC or HPLC. Individual stock standard solutions (generally containing $1000 \mu \mathrm{g} / \mathrm{ml}$ in ethyl acetate) were prepared from the reference standard materials. For the multiresidue experiments, mixed working standard solutions in acetone were prepared for each pesticide by serial dilution of the stock standard and were used as a spiking solution as well.

Chemicals: Phosphate buffer ( $\mathrm{pH} 7.0,1 \mathrm{~mol} / \mathrm{l})$; $\mathrm{K}_{2} \mathrm{HPO}_{4} 105 \mathrm{~g}$ and $\mathrm{KH}_{2} \mathrm{PO}_{4} 61 \mathrm{~g}$ were dissolved in distilled water and the $\mathrm{pH}$ was adjusted to 7 by addition of $1 \mathrm{~mol} / \mathrm{l} \mathrm{HCl}$ and $\mathrm{NaOH}$ solution. Mini column, strong anion exchange/primary-secondary amine combination (SAX/PSA) was obtained from Varian Inc. (CA, U.S.A., No. 1225-6061). All chemicals and solvents were a special grade for pesticide residue analysis.

Samples: broccoli, asparagus, carrot, spinach, burdock, matsutake mushroom, cauliflower, orange, soybean, sesame and millet were purchased from local retailers.

\section{Sample Preparation}

\section{Extraction:}

Vegetables and Fruits: Samples were homogenized, and an aliquot of $20 \mathrm{~g}$ was placed in a blender. Eighty $\mathrm{ml}$ of ethyl acetate and anhydrous sodium sulfate 50-100 $\mathrm{g}$ were added and the samples were blended for $3 \mathrm{~min}$. The extract was decanted into a round bottom flask through a filter paper No. 5. The residue was then rehomogenized with $50 \mathrm{ml}$ of ethyl acetate and filtered again and the filters were combined. These were concentrated to a few milliliters in a rotary evaporator at a water bath temperature of $40^{\circ} \mathrm{C}$. After the solvent had been removed by a gentle stream of nitrogen, the residue was dissolved and adjusted to $5 \mathrm{ml}$ with acetone- $n$-hexane $(30+70)$ solution.

Cereals and Seeds: Samples were ground, and an aliquot of $10 \mathrm{~g}$ was placed in a blender. One hundred $\mathrm{ml}$ of $35 \%(\mathrm{v} / \mathrm{v})$ water in acetonitrile was added, and the sample was blended for $3 \mathrm{~min}$. The extract was decanted into a reparatory funnel through a filter paper No. 5 . The residue was then washed with $30 \mathrm{ml}$ of acetonitrile and filtered again and the filters were combined. Seven grams of sodium chloride and $10 \mathrm{ml}$ of phosphate buffer (pH 7.0, $1 \mathrm{~mol} /$ 1) were added and shaken for $5 \mathrm{~min}$, and then allowed to stand for $15 \mathrm{~min}$. The water portion was discarded. The acetonitrile layer was collected and the solvent was removed by a rotary evaporator at $40^{\circ} \mathrm{C}$. Thirty $\mathrm{ml}$ of ethyl acetate and anhydrous sodium sulfate was added to the residue and the residue was dissolved using an ultrasonic machine, then filtered though a filter paper No. 5 . The filtrates were combined. The solvent was concentrated to a few milliliters in a rotary evaporator and was removed by a gentle stream of nitrogen. Five $\mathrm{ml}$ of acetonitrile saturated with $n$-hexane and $1.5 \mathrm{ml}$ of $n$-hexane saturated with acetonitrile were added and shaken for $5 \mathrm{~min}$. After removal of the $n$-hexane, the acetonitrile layer was concentrated. The residue was dissolved and adjusted to $5 \mathrm{ml}$ with acetone- $n$ hexane $(30+70)$ solution. The sample was centrifuged at $3000 \mathrm{rpm}$ for $5 \mathrm{~min}$ to separate the precipitation.

Cleanup: A mini column (SAX/PSA) conditioned with $5 \mathrm{ml}$ of acetone- $n$-hexane $(30+70)$ solution. Two milliliters of the extraction solution for vegetables, fruits and $4 \mathrm{ml}$ of extraction solution for cereals and seeds were transferred to a mini column, respectively. Pesticides were eluted from the column with $5 \mathrm{ml}$ of acetone- $n$-hexane $(30+70)$ solution. After the elute was concentrated with a stream of nitrogen at a water bath temperature of $40^{\circ} \mathrm{C}$, the volume was adjusted to $2 \mathrm{ml}$ for all crops with acetone- $n$-hexane $(30+70)$. The solution was used for $\mathrm{GC}$ analysis.

Recovery Test: Recovery of the 186 pesticides in broccoli, orange, asparagus, carrot, spinach, burdock, matsutake mushroom, cauliflower soybean, sesame and millet was assessed in three separate tests by fortification of each pesticide working solution to each sample. The pesticide concentration in the spiked sample was $0.1 \mathrm{mg} / \mathrm{kg}$. The spiked sample was then prepared in the manner described in Sample Preparation.

\section{Analysis of Pesticides}

GC Analysis: The prepared sample solutions were analyzed by GC (Agilent Technologies, U.S.A.) equipped with a flame photometric detector (FPD) in $\mathrm{P}$ mode, nitrogen-phosphorous detector (NPD) and ${ }^{63} \mathrm{Ni}$ electron capture detector (ECD). The FPD-GC, 

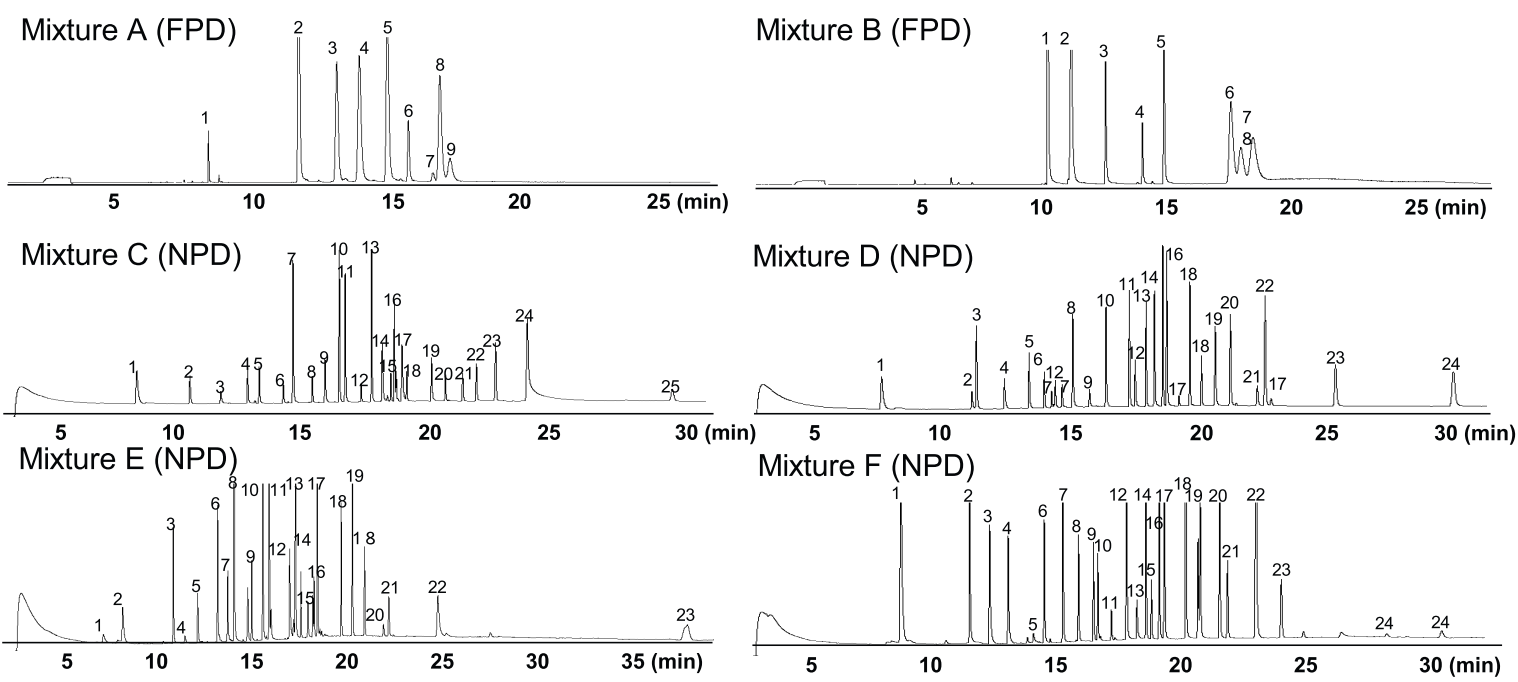

Mixture $\mathrm{G}(\mathrm{ECD})$
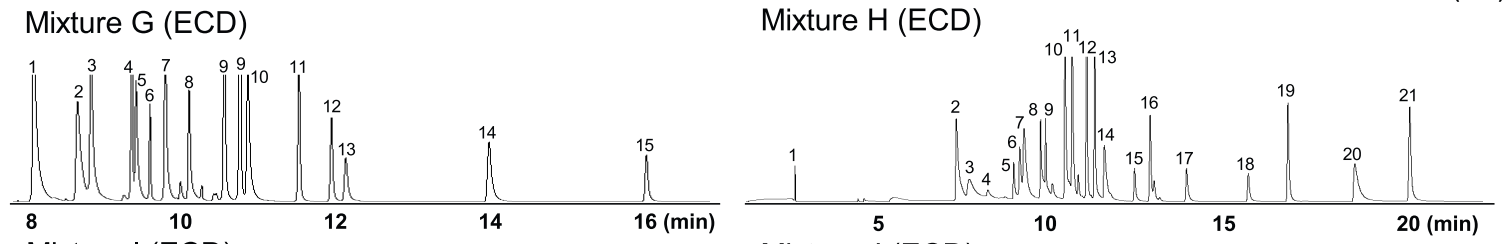

Mixture I (ECD)
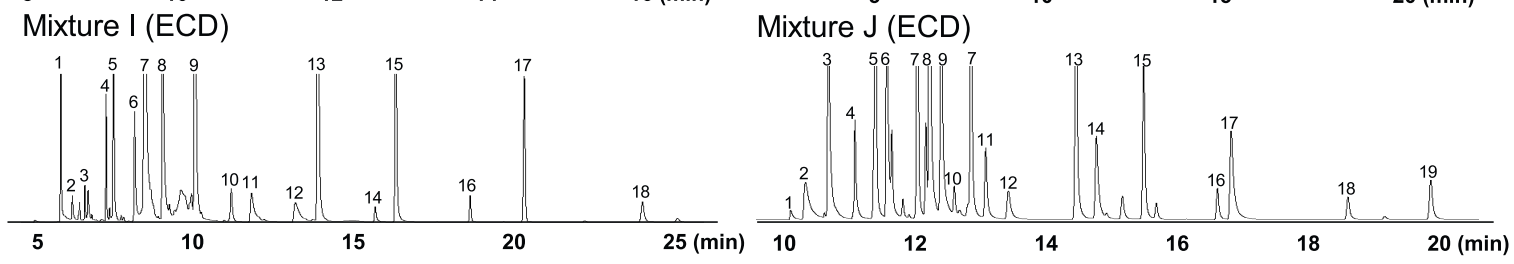

Fig. 1. Typical Gas Chromatograms of Mixed Standard Solution for 186 Pesticides

Mixture A and B, mixture C-F and mixture G-J were measured by FPD-GC, NPD-GC and ECD-GC, receptivity. Concentration of each pesticide was $1.0 \mu \mathrm{g} / \mathrm{ml}$. Peaks: (Mixture A): 1, Azamethiphos; 2, Phoxim; 3, Disulfoton; 4, Bromophos; 5, Tribuphos; 6, Fenamiphos; 7, Vamidothion; 8, Pyridafenthion; 9, Azinphos methyl, (Mixture B): 1, Mevinphos; 2, Terbufos; 3, Omethoate; 4, Phosphamidon; 5, Isoprothiolane; 6, Piperophos; 7, Anilofos; 8, Pyrazophos, (Mixture C): 1, EPTC; 2, Propham; 3, Cinosulfuron; 4, Propachlor; 5, Diphenylamine; 6, Propyzamide; 7, Atrazine; 8, Bromobutide; 9, Cyromazine; 10, Ametryn; 11, Triadimefon; 12, Zoxamide; 13, Dimethametryn; 14, Paclobutrazol; 15, Iprovalicarb; 16, Fenothiocarb; 17, Uniconazole P; 18, Flamprop-methyl; 19, Fludioxonil; 20, Benalaxyl; 21, Picolinafen; 22, Pyributicarb; 23, Furathiocarb; 24, Phosmet; 25, Cafenstrole; (Mixture D): 1, Allidochlor; 2, Triflusulfuron-methyl; 3, Molinate; 4, Pencycuron; 5, Promecarb; 6, Bendiocarb; 7, Clomazone; 8, Simeconazole; 9, Terbacil; 10, Tetraconazole; 11, Penconazol; 12, Bromacil; 13, Diphenamid; 14, Hexaconazole; 15, Buprofezin; 16, Flusilazole; 17, Tepraloxydim; 18, Metominostrobin (E); 18', Metominostrobin (Z); 19, Tebuconazole; 20, Norflurazon; 21, Fenoxycarb; 22, Amitraz; 23, Fenarimol; 24, Fenbuconazole, (Mixture E): 1, Diuron; 2, Prosulfuron; 3, Trifluralin; 4, Captan; 5, Chlorpropham; 6, Spiroxamine; 7, Ethoxyquin; 8, Simazine; 9, Dimethenamid; 10, Prometryn; 11, Terbutryn; 12, Triadimenol; 13, Cyanazine; 14, Pretilachlor; 15, Ethychlozate; 16, Imazamethabenz-methyl ester; 17, Flutriafol; 18, Pyriminobac-methyl (Z); 19, Carbosulfan; 18', Pyriminobac-methyl (E); 20, Nicosulfuron; 21, Cyhalofop-butyl; 22, Bitertanol; 23, Difenoconazole, (Mixture F): 1, Propamocarb; 2, Benfuracarb; 3, XMC (Macbal); 4, Propoxur; 5, Phenmedipham; 6, Tri-allate; 7, Fenpropimorph; 8, Alachlor; 9, Metalaxyl; 10, Diethofencarb; 11, Methiocarb; 12, Cyprodinil; 13, Quinoclamine (CAN); 14, Dimepiperate; 15, Flutolani; 16, Napropamide; 17, Bupirimate; 18, Azaconazole; 19, Propiconazole; 20, Halosulfuron methyl; 21, Etoxazole; 22, Hexazinone; 23, Pyriproxyfen; 24, Cycloxydim, (Mixture G): 1 Tecnazene; 2, Dicloran; 3 , Quintozene; 4, Dithiopyr; 5, Fenchlorphos; 6, Thiazopyr; 7, Chlorthal-Dimethyl; 8, Fipronil; 9, trans-Chlordane; 10, sis-Chlordane; 11, Oxyfluorfen; 12, Fluacrypyrim; 13, Trifloxystrobin; 14, Pyraflufen-ethyl; 15, Clomeprop; 16, Butafenacil, (Mixture H): 1, Fluroxypyrl 2, Hexaflumuron; 3, Triflumuron; 4, Fluoroimide; 5, Flufenoxuron; 6, Nitenpyram; 7, Chlorothalonil; 8, Chlorfluazuron; 9, Metolachlor; 10, Dicofol; 11, Diclocymet; 12, Oxadiazon; 13, Cyflufenamid; 14, Metamitron; 15, Clodinafop-propargyl; 16, Diclofop-methyl; 17, Cloquintocet-mexyl; 18, Iodosulfuron-methyl; 19, Etobenzanide; 20, Thiacloprid; 21, Azoxystrobin, (Mixture I): 1, Ethalfluralin; 2, Furilazole; 3, Benoxacor; 4, Acetochlor; 5, Dichlofluanid; 6, Isoxaflutole; 7, Fthalide; 8, Hexythiazox; 9, Fenoxanil; 10, Fluazinam; 11, Chloridazon; 12, Acetamiprid; 13, Tetradifon; 14, Pyraclostrobin; 15, Fluquinconazole; 16, Pyrimidifen; 17, Indoxacarb; 18, Fluthiacet-methyl, (Mixture J): 1, Dimethipin; 2, Pyrazosulfuron-ethy; 3, Vinclozolin; 4, Nitrothal-isopropyl; 5, Allethrin; 6, Triflumizole; 7, $\alpha$-Endosulfan; 8, Thifluzamide; 9, Diclobutrazol; 10, Chlorbenzilate; 7', $\beta$-Endosulfan; 11, Carfentrazone-ethyl; 12, Quinoxyfen; 13, Bromopropylate; 14, Rimsulfuron; 15, Lactofen; 16, Pyridaben; 17, Prochloraz; 18, Flumioxazin; 19, Flumiclorac-pentyl.

NPD-GC and ECD-GC were used in capillary columns: DB-210 (30 $\mathrm{m} \times 0.25 \mu \mathrm{m}$ film thickness), DB$17(30 \mathrm{~m} \times 0.25 \mu \mathrm{m}$ film thickness), and DB-5 (30 m $\times 0.25 \mu \mathrm{m}$ film thickness), all from Agilent Tech- nologies (California, U.S.A.). General operating conditions were as follows: inlet temperature $250^{\circ} \mathrm{C}$, detector temperature $280^{\circ} \mathrm{C}$, column temperature for the DB- 210 column-initially $60^{\circ} \mathrm{C}$ for $2 \mathrm{~min}$, pro- 
The 128 pesticides recovered range in $50-150 \%$
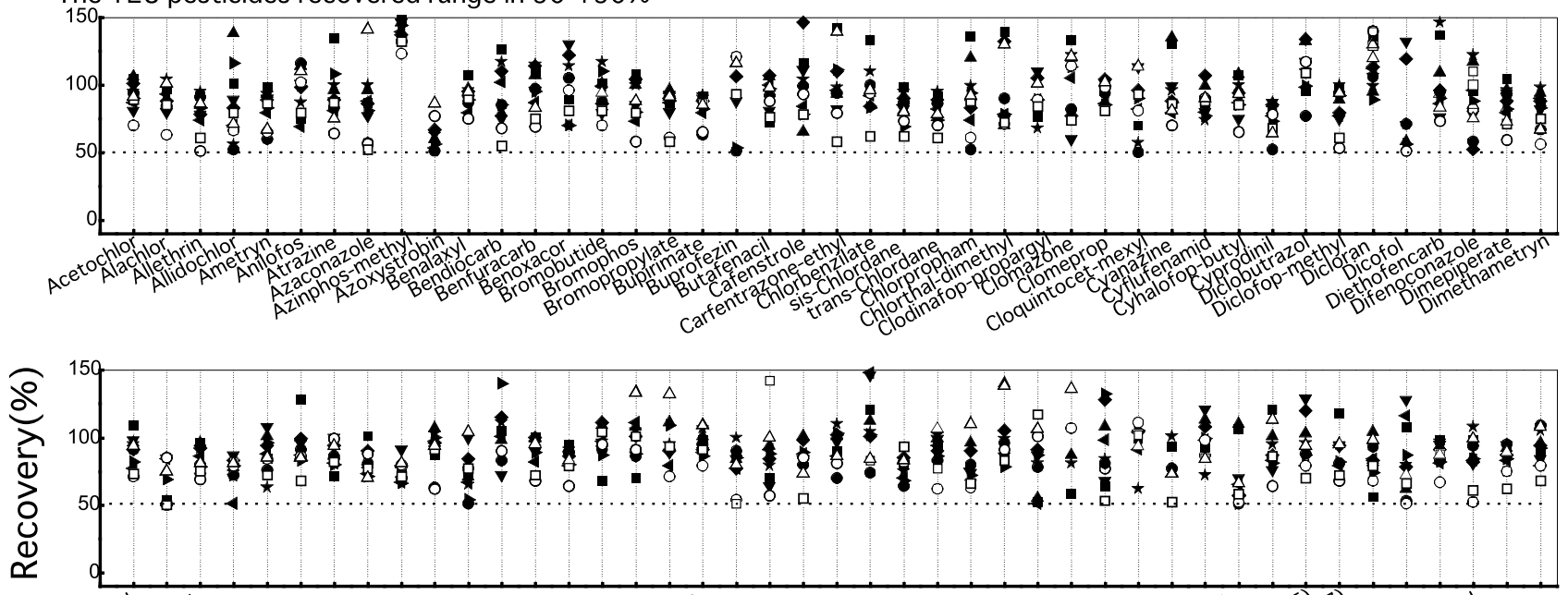

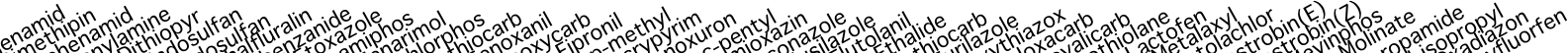

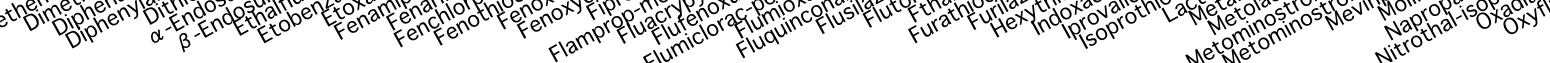
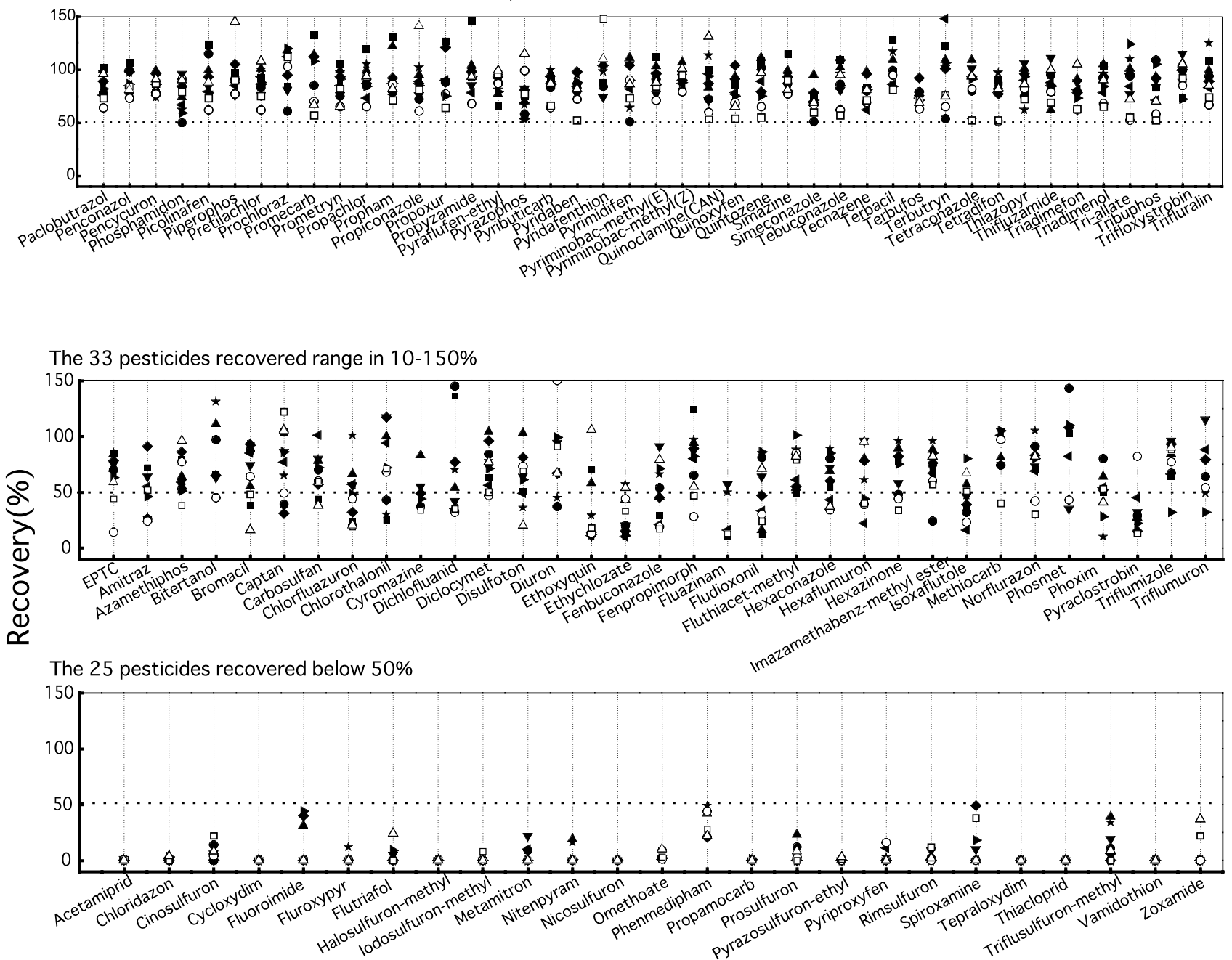

Fig. 2. Recovery of 186 Pesticides Obtained for Eleven Agricultural Products Using the Proposed Screening Methods for VegetableFruits and Cereals-Seeds

Each pesticide was spiked at $0.1 \mathrm{mg} / \mathrm{kg}$ level as well as analysis of one blank extract. Values are average of three replicate determinations. Orange, $\Delta$ Asparagus, $\square$ Broccoli, $\nabla$ Carrot, $\diamond$ Spinach, 4 Burdock, $\downarrow$ Matsutake mushroom, $\star$ Cauliflower, $\bigcirc$ Soybean, $\square$ Sesame, $\triangle$ Millet. 
Table 1. Limit of Quantitation (LOQ) of GC and GC/MS and Conformation Ions of GC/MS for Pesticide-Monitoring Programme by the Proposed Screening Methods

\begin{tabular}{|c|c|c|c|c|c|c|c|c|c|c|c|c|c|}
\hline \multirow{3}{*}{$\begin{array}{r}\text { No. } \\
1\end{array}$} & \multirow{3}{*}{$\begin{array}{l}\text { Pesticide }^{a)} \\
\text { Acetochlor }\end{array}$} & \multicolumn{2}{|c|}{ LOQ (ppm) } & \multirow{2}{*}{\multicolumn{3}{|c|}{$\begin{array}{c}\text { GC/MS confirmation } \\
\text { ions, } m / z\end{array}$}} & \multirow{3}{*}{$\begin{array}{r}\text { No. } \\
51\end{array}$} & \multirow{3}{*}{$\begin{array}{l}\text { Pesticide }^{a)} \\
\text { Etoxazole }\end{array}$} & \multicolumn{2}{|c|}{ LOQ (ppm) } & \multirow{2}{*}{\multicolumn{3}{|c|}{$\begin{array}{c}\text { GC/MS confirmation } \\
\text { ions, } m / z\end{array}$}} \\
\hline & & \multirow{2}{*}{$\begin{array}{l}\mathrm{GC} \\
0.1\end{array}$} & \multirow{2}{*}{$\begin{array}{c}\text { GC/MS } \\
0.2\end{array}$} & & & & & & GC & GC/MS & & & \\
\hline & & & & 223 & 174 & 162 & & & 0.1 & 0.1 & 359 & 330 & 300 \\
\hline 2 & Alachlor & 0.01 & 0.01 & 269 & 188 & 160 & 52 & Fenamiphos & 0.01 & 0.01 & 303 & 288 & 260 \\
\hline 3 & Allethrin & 0.05 & 0.05 & 168 & 136 & 123 & 53 & Fenarimol & 0.1 & 0.1 & 330 & 251 & 219 \\
\hline 4 & Allidochlor & 0.1 & 0.1 & 173 & 138 & 132 & 54 & Fenchlorphos & 0.01 & 0.01 & 287 & 167 & 109 \\
\hline 5 & Ametryn & 0.03 & 0.03 & 227 & 212 & 185 & 55 & Fenothiocarb & 0.1 & 0.1 & 253 & 160 & 72 \\
\hline 6 & Anilofos & 0.01 & 0.01 & 334 & 228 & 226 & 56 & Fenoxanil & 0.05 & 0.05 & 328 & 293 & 189 \\
\hline 7 & Atrazine & 0.03 & 0.03 & 215 & 200 & 173 & 57 & Fenoxycarb & 0.1 & 0.1 & 301 & 255 & 186 \\
\hline 8 & Azaconazole & 0.1 & 0.1 & 217 & 173 & 145 & 58 & Fipronil & 0.01 & 0.01 & 367 & 351 & 213 \\
\hline 9 & Azinphos-methyl & 0.05 & 0.05 & 160 & 132 & 125 & 59 & Flamprop-methyl & 0.1 & 0.1 & 335 & 276 & 230 \\
\hline 10 & Azoxystrobin & 0.01 & 0.01 & 403 & 388 & 344 & 60 & Fluacrypyrim & 0.01 & 0.01 & 426 & 320 & 204 \\
\hline 11 & Benalaxyl & 0.1 & 0.1 & 325 & 266 & 206 & 61 & Flufenoxuron & 0.02 & 0.02 & 331 & 268 & 227 \\
\hline 12 & Bendiocarb & 0.02 & 0.02 & 223 & 166 & 151 & 62 & Flumiclorac-pentyl & 0.1 & 0.1 & 423 & 353 & 318 \\
\hline 13 & Benfluralin & 0.1 & 0.1 & 292 & 276 & 264 & 63 & Flumioxazin & 0.02 & 0.02 & 354 & 287 & 259 \\
\hline 14 & Benoxazol & 0.1 & 0.2 & 259 & 176 & 120 & 64 & Fluquinconazole & 0.02 & 0.02 & 375 & 340 & 298 \\
\hline 15 & Bromobutide & 0.1 & 0.2 & 296 & 232 & 119 & 65 & Flusilazole & 0.01 & 0.01 & 315 & 233 & 206 \\
\hline 16 & Bromophos & 0.01 & 0.01 & 331 & 213 & 125 & 66 & Flutolanil & 0.1 & 0.2 & 323 & 281 & 173 \\
\hline 17 & Bromopropylate & 0.02 & 0.02 & 341 & 183 & 157 & 67 & Fthalide & 0.1 & 0.1 & 272 & 243 & 215 \\
\hline 18 & Bupirimate & 0.05 & 0.05 & 316 & 273 & 208 & 68 & Furathiocarb & 0.05 & 0.05 & 382 & 325 & 194 \\
\hline 19 & Buprofezin & 0.05 & 0.05 & 305 & 249 & 172 & 69 & Furilazole & 0.1 & 0.2 & 262 & 220 & 181 \\
\hline 20 & Butafenacil & 0.03 & 0.03 & 331 & 180 & 124 & 70 & Hexythiazox & 0.1 & 0.1 & 227 & 184 & 156 \\
\hline 21 & Cafenstrole & 0.1 & 0.1 & 188 & 119 & 100 & 71 & Indoxacarb & 0.1 & 0.2 & 366 & 264 & 218 \\
\hline 22 & Carfentrazone-ethyl & 0.05 & 0.05 & 411 & 376 & 340 & 72 & Iprovalicarb & 0.1 & 0.2 & 260 & 158 & 134 \\
\hline 23 & Chlorbenzilate & 0.1 & 0.1 & 251 & 139 & 111 & 73 & Isoprothiolane & 0.02 & 0.02 & 290 & 231 & 204 \\
\hline 24 & Chlordane & 0.02 & 0.02 & 410 & 373 & 272 & 74 & Lactofen & 0.05 & 0.05 & 461 & 344 & 223 \\
\hline 25 & Chlorpropham & 0.05 & 0.05 & 213 & 171 & 127 & 75 & Metalaxyl & 0.1 & 0.1 & 249 & 234 & 220 \\
\hline 26 & Chlorthal-dimethyl & 0.02 & 0.02 & 322 & 301 & 221 & 76 & Metolachlor & 0.02 & 0.02 & 238 & 162 & 146 \\
\hline 27 & Clodinafop-propargyl & 0.1 & 0.1 & 349 & 266 & 238 & 77 & Metominostrobin & 0.1 & 0.2 & 284 & 238 & 196 \\
\hline 28 & Clomazone & 0.1 & 0.4 & 204 & 138 & 125 & 78 & Mevinphos & 0.05 & 0.05 & 192 & 164 & 127 \\
\hline 29 & Clomeprop & 0.05 & 0.05 & 323 & 288 & 203 & 79 & Molinate & 0.05 & 0.05 & 187 & 126 & 98 \\
\hline 30 & Cloquintocet-mexyl & 0.1 & 0.1 & 220 & 192 & 163 & 80 & Napropamide & 0.05 & 0.05 & 271 & 171 & 128 \\
\hline 31 & Cyanazine & 0.02 & 0.02 & 240 & 225 & 198 & 81 & Nitrothal-isopropyl & 0.05 & 0.05 & 254 & 212 & 136 \\
\hline 32 & Cyflufenamid & 0.1 & 0.1 & 412 & 321 & 294 & 82 & Oxadiazon & 0.02 & 0.02 & 344 & 302 & 258 \\
\hline 33 & Cyhalofop-butyl & 0.1 & 0.1 & 357 & 256 & 229 & 83 & Oxyfluorfen & 0.05 & 0.05 & 361 & 317 & 300 \\
\hline 34 & Cyprodinil & 0.02 & 0.02 & 225 & 186 & 128 & 84 & Paclobutrazol & 0.01 & 0.01 & 236 & 167 & 125 \\
\hline 35 & Diclobutrazol & 0.05 & 0.05 & 270 & 201 & 159 & 85 & Penconazol & 0.05 & 0.05 & 248 & 213 & 159 \\
\hline 36 & Diclofop-methyl & 0.05 & 0.05 & 340 & 281 & 253 & 86 & Pencycuron & 0.1 & 0.1 & 209 & 180 & 125 \\
\hline 37 & Dicloran & 0.1 & 0.1 & 210 & 206 & 176 & 87 & Phosphamidon & 0.01 & 0.01 & 264 & 193 & 127 \\
\hline 38 & Dicofol & 0.1 & 0.2 & 250 & 215 & 139 & 88 & Picolinafen & 0.1 & 0.1 & 376 & 266 & 238 \\
\hline 39 & Diethofencarb & 0.1 & 0.1 & 267 & 225 & 196 & 89 & Piperophos & 0.03 & 0.03 & 320 & 140 & 122 \\
\hline 40 & Difenoconazole & 0.02 & 0.02 & 325 & 323 & 152 & 90 & Pretilachlor & 0.1 & 0.1 & 262 & 238 & 225 \\
\hline 41 & Dimepiperate & 0.1 & 0.1 & 263 & 145 & 119 & 91 & Prochloraz & 0.05 & 0.05 & 308 & 266 & 180 \\
\hline 42 & Dimethametryn & 0.1 & 0.1 & 255 & 240 & 212 & 92 & Promecarb & 0.1 & 0.1 & 207 & 150 & 135 \\
\hline 43 & Dimethenamid & 0.01 & 0.01 & 230 & 203 & 154 & 93 & Prometryn & 0.05 & 0.05 & 241 & 226 & 184 \\
\hline 44 & Dimethipin & 0.05 & 0.05 & 210 & 124 & 118 & 94 & Propachlor & 0.1 & 0.5 & 211 & 196 & 176 \\
\hline 45 & Diphenamid & 0.05 & 0.05 & 239 & 167 & 152 & 95 & Propham & 0.1 & 0.1 & 179 & 137 & 120 \\
\hline 46 & Diphenylamine & 0.05 & 0.05 & 169 & 141 & 83 & 96 & Propiconazole & 0.05 & 0.05 & 259 & 191 & 173 \\
\hline 47 & Dithiopyr & 0.05 & 0.05 & 354 & 306 & 286 & 97 & Propoxur & 0.1 & 0.1 & 152 & 110 & 81 \\
\hline 48 & Endosulfan & 0.05 & 0.05 & 339 & 277 & 265 & 98 & Propyzamide & 0.1 & 0.2 & 255 & 240 & 173 \\
\hline 49 & Ethalfluralin & 0.1 & 0.1 & 333 & 316 & 292 & 99 & Pyraflufen-ethyl & 0.05 & 0.05 & 412 & 349 & 339 \\
\hline 50 & Etobenzanide & 0.1 & 0.1 & 339 & 304 & 179 & 100 & Pyrazophos & 0.01 & 0.01 & 373 & 265 & 232 \\
\hline
\end{tabular}

a) Validated pesticides in this study. $b$ ) Validated pesticides in our previous study. 
Table 1. Continued

\begin{tabular}{|c|c|c|c|c|c|c|c|c|c|c|c|c|c|}
\hline \multirow{3}{*}{$\begin{array}{l}\text { No. } \\
101\end{array}$} & \multirow{3}{*}{$\begin{array}{l}\text { Pesticide }^{a)} \\
\text { Pyributicarb }\end{array}$} & \multicolumn{2}{|c|}{ LOQ (ppm) } & \multirow{2}{*}{\multicolumn{3}{|c|}{$\begin{array}{c}\text { GC/MS confirmation } \\
\text { ions, } m / z\end{array}$}} & \multirow{3}{*}{$\begin{array}{l}\text { No. } \\
129\end{array}$} & \multirow{3}{*}{$\begin{array}{l}\text { Pesticide }^{b)} \\
\text { Acrinathrin }\end{array}$} & \multicolumn{2}{|c|}{ LOQ (ppm) } & \multirow{2}{*}{\multicolumn{3}{|c|}{$\begin{array}{c}\text { GC/MS confirmation } \\
\text { ions, } \mathrm{m} / z\end{array}$}} \\
\hline & & \multirow{2}{*}{$\begin{array}{l}\text { GC } \\
0.1\end{array}$} & \multirow{2}{*}{$\begin{array}{c}\text { GC/MS } \\
0.1\end{array}$} & & & & & & \multirow{2}{*}{$\begin{array}{l}\mathrm{GC} \\
0.1\end{array}$} & \multirow{2}{*}{$\begin{array}{c}\text { GC/MS } \\
0.1\end{array}$} & & & \\
\hline & & & & 181 & 165 & 108 & & & & & 289 & 208 & 181 \\
\hline 102 & Pyridaben & 0.05 & 0.05 & 364 & 309 & 147 & 130 & Bifenox & 0.05 & 0.05 & 341 & 310 & 189 \\
\hline 103 & Pyridafenthion & 0.01 & 0.01 & 340 & 199 & 188 & 131 & Bifenthrin & 0.05 & 0.05 & 181 & 166 & 152 \\
\hline 104 & Pyrimidifen & 0.1 & 0.1 & 220 & 184 & 161 & 132 & Buthchlor & 0.1 & 0.1 & 311 & 237 & 176 \\
\hline 105 & Pyriminobac-methyl & 0.1 & 0.1 & 330 & 302 & 256 & 133 & Butamifos & 0.05 & 0.05 & 286 & 232 & 200 \\
\hline 106 & Quinoclamine(CAN) & 0.1 & 0.1 & 207 & 172 & 144 & 134 & Cadusafos & 0.01 & 0.01 & 270 & 214 & 159 \\
\hline 107 & Quinoxyfen & 0.1 & 0.1 & 307 & 272 & 237 & 135 & Carbofuran & 0.1 & 0.1 & 221 & 164 & 149 \\
\hline 108 & Quintozene (PCNB) & 0.1 & 0.1 & 295 & 265 & 249 & 136 & Chlorfenvinphos & 0.02 & 0.02 & 323 & 295 & 267 \\
\hline 109 & Simazine (CAT) & 0.1 & 0.1 & 201 & 186 & 128 & 137 & Chlorphenapyr & 0.05 & 0.05 & 408 & 363 & 328 \\
\hline 110 & Simeconazole & 0.05 & 0.05 & 278 & 211 & 195 & 138 & Chlorpyrifos & 0.01 & 0.01 & 314 & 286 & 258 \\
\hline 111 & Tebuconazole & 0.05 & 0.05 & 250 & 163 & 125 & 139 & Chlorpyrifos-methyl & 0.01 & 0.01 & 323 & 286 & 125 \\
\hline 112 & Tecnazene & 0.1 & 0.1 & 261 & 215 & 203 & 140 & Cyanophos & 0.02 & 0.02 & 243 & 180 & 125 \\
\hline 113 & Terbacil & 0.04 & 0.04 & 216 & 161 & 117 & 141 & Cyfluthrin & 0.05 & 0.05 & 226 & 206 & 163 \\
\hline 114 & Terbufos & 0.005 & 0.005 & 288 & 231 & 186 & 142 & Cyhalothrin & 0.04 & 0.04 & 449 & 208 & 197 \\
\hline 115 & Terbutryn & 0.02 & 0.02 & 241 & 226 & 185 & 143 & Cypermethrin & 0.05 & 0.05 & 209 & 181 & 163 \\
\hline 116 & Tetraconazole & 0.05 & 0.05 & 336 & 267 & 171 & 144 & Deltamethrin & 0.01 & 0.01 & 253 & 209 & 181 \\
\hline 117 & Tetradifon & 0.01 & 0.01 & 359 & 229 & 159 & 145 & Diazinon & 0.01 & 0.01 & 304 & 199 & 179 \\
\hline 118 & Thiazopyr & 0.1 & 0.1 & 396 & 381 & 327 & 146 & Dichlofenthion & 0.01 & 0.01 & 279 & 251 & 223 \\
\hline 119 & Thifluzamide & 0.1 & 0.1 & 447 & 429 & 194 & 147 & Dichlorvos(DDVP) & 0.02 & 0.02 & 220 & 185 & 145 \\
\hline 120 & Triadimefon & 0.1 & 0.2 & 208 & 181 & 128 & 148 & Dimethoate & 0.1 & 0.1 & 229 & 143 & 125 \\
\hline 121 & Triadimenol & 0.1 & 0.2 & 168 & 128 & 112 & 149 & Dimethylvinphos & 0.04 & 0.04 & 295 & 204 & 170 \\
\hline 122 & Tri-allate & 0.1 & 0.1 & 268 & 143 & 128 & 150 & Edifenphos & 0.02 & 0.02 & 310 & 218 & 201 \\
\hline 123 & Tribuphos & 0.05 & 0.05 & 314 & 258 & 202 & 151 & EPN & 0.02 & 0.02 & 323 & 185 & 157 \\
\hline 124 & Trifloxystrobin & 0.02 & 0.02 & 222 & 206 & 187 & 152 & Esprocarb & 0.1 & 0.1 & 265 & 222 & 162 \\
\hline 125 & Trifluralin & 0.05 & 0.05 & 335 & 306 & 264 & 153 & Ethion & 0.01 & 0.01 & 384 & 231 & 153 \\
\hline 126 & Uniconazole $\mathrm{P}$ & 0.1 & 0.1 & 234 & 165 & 131 & 154 & Ethoprophos & 0.005 & 0.005 & 242 & 200 & 158 \\
\hline 127 & Vinclozolin & 0.05 & 0.05 & 285 & 212 & 198 & 155 & Etrimfos & 0.01 & 0.01 & 292 & 277 & 181 \\
\hline \multirow[t]{9}{*}{128} & XMC (Macbal) & 0.1 & 0.1 & 179 & 122 & 107 & 156 & Fenitrothion & 0.01 & 0.01 & 277 & 260 & 125 \\
\hline & & & & & & & 157 & Fenobucarb & 0.1 & 0.1 & 207 & 150 & 121 \\
\hline & & & & & & & 158 & Fenopropathrin & 0.01 & 0.01 & 349 & 265 & 181 \\
\hline & & & & & & & 159 & Fensulfothion & 0.02 & 0.02 & 308 & 293 & 141 \\
\hline & & & & & & & 160 & Fenthion & 0.01 & 0.01 & 278 & 169 & 153 \\
\hline & & & & & & & 161 & Fenvalerate & 0.05 & 0.05 & 419 & 225 & 181 \\
\hline & & & & & & & 162 & Flucythrinate & 0.05 & 0.05 & 451 & 225 & 199 \\
\hline & & & & & & & 163 & Fluvalinate & 0.01 & 0.01 & 502 & 250 & 208 \\
\hline & & & & & & & 164 & Fonofos & 0.01 & 0.01 & 246 & 137 & 109 \\
\hline
\end{tabular}

grammed to $250^{\circ} \mathrm{C}$ at $15^{\circ} \mathrm{C} / \mathrm{min}$, finally $250^{\circ} \mathrm{C}$ for $5 \mathrm{~min}$; for the DB-17 column-initially $100^{\circ} \mathrm{C}$ for $2 \mathrm{~min}$, heated to $270^{\circ} \mathrm{C}$ at $10^{\circ} \mathrm{C} / \mathrm{min}$, finally $250^{\circ} \mathrm{C}$ for $6 \mathrm{~min}$; for the DB-5 column-initially $150^{\circ} \mathrm{C}$ for $2 \mathrm{~min}$, programmed to $260^{\circ} \mathrm{C}$ at $40^{\circ} \mathrm{C} / \mathrm{min}$, finally $260^{\circ} \mathrm{C}$ for $6 \mathrm{~min}$; carrier gas (helium) flow $2 \mathrm{ml} / \mathrm{min}$, sample injection volume $2 \mu \mathrm{l}$; injection mode, splitless.

GC/MS Analysis: A mass chromatogram was obtained on a GC/MS (Agilent Technologies, U.S.A.) equipped with a quadrupole mass spectrometer. The GC/MS was used in the above capillary column DB-5. General operating conditions were as follows: inlet temperature $250^{\circ} \mathrm{C}$; transfer line temperature $280^{\circ} \mathrm{C}$, ionization temperature, $150^{\circ} \mathrm{C}$, column temperature initially $60^{\circ} \mathrm{C}$ for $1 \mathrm{~min}$, programmed to $280^{\circ} \mathrm{C}$ at $10^{\circ} \mathrm{C} / \mathrm{min}$, finally 280 for $7 \mathrm{~min}$; mass spectrometer setting; electro ionization potential $70 \mathrm{eV}$, sample injection volume $2 \mu \mathrm{l}$; injection mode, splitless. 
Table 1. Continued

\begin{tabular}{|c|c|c|c|c|c|c|}
\hline \multirow{3}{*}{$\begin{array}{l}\text { No. } \\
165\end{array}$} & \multirow{3}{*}{$\begin{array}{l}\text { Pesticide }^{b)} \\
\text { Fosthiazate }\end{array}$} & \multicolumn{2}{|c|}{$\overline{~ L O Q ~(p p m) ~}$} & \multirow{2}{*}{\multicolumn{3}{|c|}{$\begin{array}{c}\text { GC/MS confirmation } \\
\text { ions, } m / z\end{array}$}} \\
\hline & & \multirow{2}{*}{$\begin{array}{l}\mathrm{GC} \\
0.02\end{array}$} & \multirow{2}{*}{$\frac{\text { GC/MS }}{0.02}$} & & & \\
\hline & & & & 283 & 227 & 195 \\
\hline 166 & Halfenprox & 0.05 & 0.05 & 478 & 263 & 183 \\
\hline 167 & Iprobenfos & 0.01 & 0.01 & 288 & 246 & 204 \\
\hline 168 & Isazophos & 0.01 & 0.01 & 285 & 257 & 161 \\
\hline 169 & Isofenphos & 0.01 & 0.01 & 255 & 213 & 185 \\
\hline 170 & Isoprocarb & 0.1 & 0.1 & 193 & 136 & 121 \\
\hline 171 & Kresoxim-methyl & 0.1 & 0.1 & 313 & 206 & 131 \\
\hline 172 & Malathion & 0.01 & 0.01 & 285 & 173 & 158 \\
\hline 173 & Mepronil & 0.1 & 0.25 & 269 & 210 & 119 \\
\hline 174 & Methacrifos & 0.01 & 0.01 & 240 & 208 & 180 \\
\hline 175 & Methamidophos & 0.05 & 0.05 & 141 & 126 & 94 \\
\hline 176 & Methidathion & 0.01 & 0.01 & 302 & 145 & 125 \\
\hline 177 & Myclobutanil & 0.1 & 0.1 & 288 & 179 & 150 \\
\hline 178 & Parathion & 0.01 & 0.01 & 291 & 186 & 139 \\
\hline 179 & Parathion-methyl & 0.01 & 0.01 & 263 & 125 & 109 \\
\hline 180 & Pendimethalin & 0.05 & 0.05 & 281 & 252 & 162 \\
\hline 181 & Permethrin & 0.05 & 0.05 & 390 & 183 & 163 \\
\hline 182 & Phenthoate & 0.01 & 0.01 & 274 & 246 & 157 \\
\hline 183 & Phorate & 0.01 & 0.01 & 260 & 231 & 121 \\
\hline 184 & Phosalone & 0.02 & 0.02 & 367 & 182 & 121 \\
\hline 185 & Pirimiphos-methyl & 0.01 & 0.01 & 305 & 290 & 276 \\
\hline 186 & Procymidone & 0.1 & 0.2 & 283 & 255 & 212 \\
\hline 187 & Profenofos & 0.02 & 0.02 & 399 & 374 & 208 \\
\hline 188 & Propaphos & 0.01 & 0.01 & 304 & 262 & 220 \\
\hline 189 & Prothiofos & 0.01 & 0.01 & 309 & 267 & 239 \\
\hline 190 & Pyraclofos & 0.05 & 0.05 & 360 & 194 & 138 \\
\hline 191 & Pyrimethanil & 0.05 & 0.05 & 198 & 184 & 158 \\
\hline 192 & Quinalphos & 0.01 & 0.01 & 298 & 241 & 146 \\
\hline 193 & Salithion & 0.01 & 0.01 & 216 & 201 & 183 \\
\hline 194 & Tebufenpyrad & 0.05 & 0.05 & 333 & 318 & 276 \\
\hline 195 & Tefluthrin & 0.1 & 0.1 & 197 & 177 & 141 \\
\hline 196 & Tetrachlorvinphos & 0.01 & 0.01 & 329 & 240 & 109 \\
\hline 197 & Thenylchlor & 0.1 & 0.1 & 323 & 288 & 127 \\
\hline 198 & Thiobencarb & 0.05 & 0.05 & 257 & 125 & 100 \\
\hline 199 & Tolclofos-methyl & 0.02 & 0.02 & 265 & 250 & 125 \\
\hline 200 & Triazophos & 0.02 & 0.02 & 313 & 285 & 257 \\
\hline
\end{tabular}

\section{RESULTS AND DISCUSSION}

\section{Pesticide Determinations by GC}

Optimal conditions for the determinations of organophosphorus, organonitrogen, organochlorine and pyrethroid pesticides were investigated. Figure 1 shows the typical gas chromatograms of standards for the 186 pesticides. Organophosphorus pesticides, organochlorine and pyrethroid pesticides, and organonitrogen pesticides were determined by FPDGC, ECD-GC, and NPD-GC, respectively. These pesticides were detected within $30 \mathrm{~min}$ except for difenoconazol. Pesticides were divided into ten groups for their complete separation in the test recovery. Adequate separation of all pesticides was achieved with good sharp peaks.

\section{Recovery Studies}

Vegetables-fruits and cereals-seeds methods were used to validate the 186 pesticides in the eleven agricultural products, as well as analysis of one blank extract. Recovery results are indicated in Fig. 2. As shown in Fig. 2, the recoveries of 128 pesticides were satisfactory (50-150\%). Three repetitive determinations of recovery achieved good reproducibility for these pesticides (RSDs: 4-21\%). All organochlorine and pyrethroid compounds in the 128 pesticides were recovered at greater than $70 \%$. The organonitrogen pesticides belonging to acetamide, acid amide, azine, benzofuran, diazine, dicarboxyimide, diphenyl ether, naphthoquinone and dinitroaniline classes also gave good recoveries $(>70 \%)$. On the other hand, the recoveries of 33 pesticides were within $10-150 \%$ and 25 pesticides were below $50 \%$ in all samples. Lower recoveries $(<50 \%)$ are thought to be due to the unique traits of these particular pesticides. Amitraz is likely to be hydrolyzed ${ }^{13,14)}$ in a sample at the extraction step. $N$-trihalomethylthio fungicides, such as captan and dichlofluanid may be degraded by a chemical reaction with thiol groups (-SH) of crop compounds during the extraction step. ${ }^{15)}$ Phoxim and carbosulfan may be decomposed by the interaction of food components at the inlet liner of GC. ${ }^{16,17)}$ The polar pesticides ethychlozate, phenmedipham, acetamiprid, chloridazon, cinosulfuron, fluoroimide, flutriafol, halosulfuron methyl, indosulfuron methyl, metamitron, nitenpyram, nicosulfuron, omethoate, phoxim, propamocarb, pyrazosulfuron-ethyl, pyriproxyfen, rimsulfuron, thiacloprid, triflusulfuron-methyl and vamidothion might not be eluted sufficiently with $30 \%$ acetone $/ n$-hexane from a SAX-PSA cartridge. Overlapping peaks interfering with target pesticides also cause poor recovery. Cycloxydim, fluroxypyr, prosulfuron, spiroxamine and zoxamide were significantly overlapped with a sample matrix of almost all agricultural products.

These results clearly demonstrate that the 128 pesticides recovered in amounts of from 50 to $150 \%$ are acceptable for screening purposes.

\section{Regulatory Sample Analysis in Quarantine Sta- tion}

The proposed methods were used for pesticide 
Table 2. Results of Monitoring of Imported Foods by Pesticide-Monitoring Programme Using Proposed Methods at the Quarantine Station in Japan during April 1 to 30, 2005

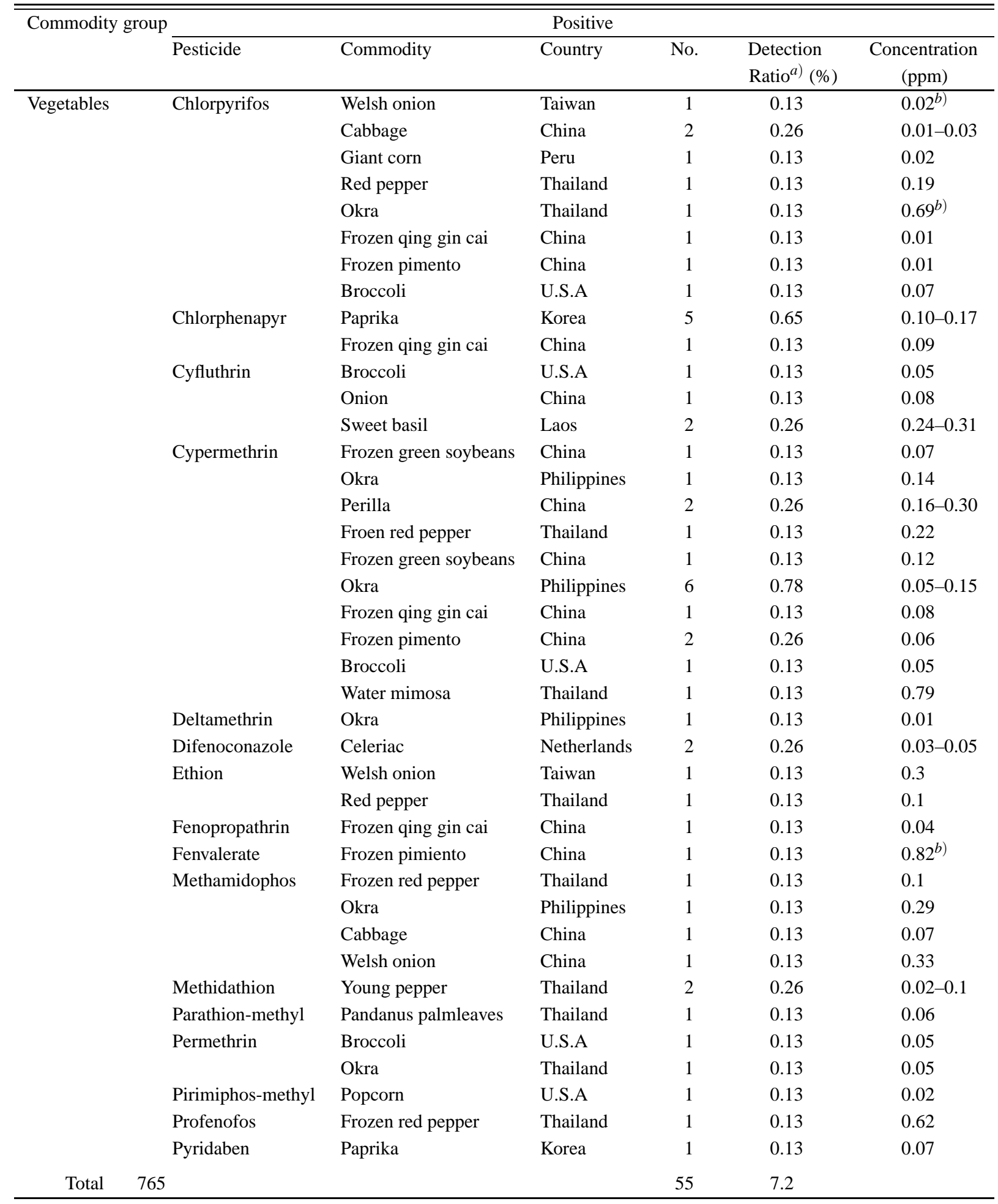

a) Number of positive samples/number of measured samples. $b$ ) Violation of Japanese maximum residue limits (MRLs).

monitoring in real imported foods at the Quarantine Station for the period April 1 to 30, 2005. During this period, 1516 samples were screened for a total of 200 pesticides including 72 which had been pre- viously validated. Table 1 lists the pesticides, limits of quantitation and GC/MS conformation ions used in the monitoring. The GC/MS [selected ion monitoring (SIM)] techniques have also been used to iden- 
Table 2. Continued

\begin{tabular}{|c|c|c|c|c|c|c|c|}
\hline \multicolumn{2}{|c|}{ Commodity group } & \multicolumn{6}{|c|}{ Positive } \\
\hline & & Pesticide & Commodity & Country & No. & $\begin{array}{l}\text { Detection } \\
\text { Ratio }^{a)}(\%)\end{array}$ & $\begin{array}{c}\text { Concentration } \\
(\mathrm{ppm})\end{array}$ \\
\hline \multirow{10}{*}{\multicolumn{2}{|c|}{ Tea }} & Chlorpyrifos & Tea & England & 1 & 2.6 & 0.01 \\
\hline & & & Tea & France & 1 & 2.6 & 0.05 \\
\hline & & Cypermethrin & Oolong tea & China & 1 & 2.6 & 0.5 \\
\hline & & DDT & Tea & England & 3 & 7.7 & $0.02-0.04$ \\
\hline & & & Tea & France & 1 & 2.6 & 0.05 \\
\hline & & Endosulfan & Tea & England & 2 & 5.1 & $0.06-0.07$ \\
\hline & & Ethion & Tea & France & 1 & 2.6 & 0.02 \\
\hline & & & Tea & Italy & 1 & 2.6 & 0.01 \\
\hline & & & Tea & England & 5 & 12.8 & $0.02-0.07$ \\
\hline & & Fenvalerate & Oolong tea & China & 1 & 2.6 & 0.8 \\
\hline Total & 39 & & & & 17 & 43.6 & \\
\hline \multirow[t]{28}{*}{ Fruits } & & Azoxystrobin & Banana & Philippines & 2 & 0.63 & $0.02-0.06$ \\
\hline & & & Mango & Philippines & 1 & 0.32 & 0.01 \\
\hline & & Bifenthrin & Frozen raspberry & U.S.A & 2 & 0.63 & $0.08-0.17$ \\
\hline & & Chlorpyrifos & Orange & U.S.A & 29 & 9.2 & $0.01-0.33$ \\
\hline & & & Banana & Philippines & 5 & 1.6 & $0.01-0.02$ \\
\hline & & & Grape & Chile & 10 & 3.2 & $0.01-0.43$ \\
\hline & & & Lemon & U.S.A & 12 & 3.8 & $0.02-0.13$ \\
\hline & & & Minneola & U.S.A & 1 & 0.32 & 0.22 \\
\hline & & & Tangerine & U.S.A & 2 & 0.63 & $0.01-0.21$ \\
\hline & & & Grapefruit & U.S.A & 1 & 0.32 & 0.01 \\
\hline & & & Persimmon & New Zealand & 1 & 0.32 & $0.02^{b)}$ \\
\hline & & Chlorphenapyr & Leaf of radish & China & 1 & 0.32 & 0.11 \\
\hline & & Cyfluthrin & Lemon & U.S.A & 1 & 0.32 & 0.09 \\
\hline & & Cypermethrin & Avocado & Mexico & 2 & 0.63 & $0.07-0.11$ \\
\hline & & Cyprodinil & Grape & Chile & 7 & 2.2 & $0.13-0.32$ \\
\hline & & & Frozen blueberry & U.S.A & 1 & 0.32 & 0.02 \\
\hline & & & Frozen raspberry & U.S.A & 2 & 0.63 & $0.03-0.04$ \\
\hline & & Deltamethrin & Orange & U.S.A & 2 & 0.63 & 0.02 \\
\hline & & & Banana & Philippines & 1 & 0.32 & 0.01 \\
\hline & & Edifenphos & Orange & U.S.A & 1 & 0.32 & 0.16 \\
\hline & & Ethion & Avocado & Mexico & 1 & 0.32 & 0.02 \\
\hline & & Fenopropathrin & Orange & U.S.A & 2 & 0.63 & $0.01-0.06$ \\
\hline & & Fenvalerate & Frozen raspberry & U.S.A & 1 & 0.32 & 0.09 \\
\hline & & Malathion & Grape & U.S.A & 1 & 0.32 & 0.02 \\
\hline & & & Orange & U.S.A & 1 & 0.32 & 0.12 \\
\hline & & & Frozen blueberry & U.S.A & 1 & 0.32 & 0.11 \\
\hline & & & Frozen raspberry & U.S.A & 1 & 0.32 & 0.02 \\
\hline & & Methidathion & Orange & U.S.A & 1 & 0.32 & 0.22 \\
\hline Total & 315 & & & & 93 & 29.5 & \\
\hline \multirow[t]{7}{*}{ Cereals } & & Chlorpyrifos-methyl & Wheat & U.S.A & 4 & 5.9 & $0.02-0.09$ \\
\hline & & & Wheat flour & Korea & 1 & 1.5 & 0.06 \\
\hline & & Cyfluthrin & Soybean & U.S.A & 1 & 1.5 & 0.06 \\
\hline & & Fenvalerate & Millet & China & 1 & 1.5 & 0.12 \\
\hline & & Malathion & Wheat & Canada & 1 & 1.5 & 0.02 \\
\hline & & & & U.S.A & 4 & 5.9 & $0.08-0.78$ \\
\hline & & & Wheat flour & Korea & 1 & 1.5 & 0.03 \\
\hline Total & 68 & & & & 13 & 19.1 & \\
\hline
\end{tabular}


Table 2. Continued

\begin{tabular}{|c|c|c|c|c|c|c|c|}
\hline \multirow{2}{*}{\multicolumn{2}{|c|}{ Commodity group }} & \multicolumn{6}{|c|}{ Positive } \\
\hline & & Pesticide & Commodity & Country & No. & $\begin{array}{l}\begin{array}{l}\text { Detection } \\
\text { Ratio }^{a)}(\%)\end{array} \\
\end{array}$ & $\begin{array}{c}\text { Concentration } \\
(\mathrm{ppm})\end{array}$ \\
\hline \multirow[t]{7}{*}{ Seeds, beans } & & Amethryn & Bean & U.S.A & 1 & 0.30 & 0.08 \\
\hline & & Chlorpyrifos & Cacao bean & Ghana & 2 & 0.61 & $0.06-0.15$ \\
\hline & & Cyfluthrin & Coffee & Guatemala & 1 & 0.30 & 0.06 \\
\hline & & Dichlorvos(DDVP) & Walnut & China & 1 & 0.30 & 0.04 \\
\hline & & Malathion & Cacao bean & Ecuador & 1 & 0.30 & 0.15 \\
\hline & & & Green bean & China & 3 & 0.91 & $0.02-0.04$ \\
\hline & & Methamidophos & Frozen kidney bean & China & 1 & 0.30 & 0.05 \\
\hline Total & 329 & & & & 10 & 3.0 & \\
\hline \multicolumn{8}{|l|}{ Grand total } \\
\hline & 1516 & & & & 188 & 12.4 & (Positive sample) \\
\hline & & & & & 4 & 0.26 & (Violation sample) \\
\hline
\end{tabular}

tify the pesticides detected by GC analysis. Since Sphon ${ }^{18)}$ reported that GC/MS must operate at least 3 diagnostic ions for identification to be confirmed with the SIM mode, three characteristic masses were chosen for each pesticide. In case the positive list system is going to be implemented, additional methods development is thought to be necessary to detect pesticides at the detection limits of $0.01 \mathrm{ppm}$ or MRLs level.

The results of monitoring are shown in Table 2. A total of 188 residues were detected in 1516 samples, corresponding to a detection rate of $12.4 \%$. The pesticides implicated were azoxystrobin, amethryn, bifenthrin, chlorphenapyr, chlorpyrifos, chlorpyrifos-methyl, cyfluthrin, cypermethrin, cyprodinil, DDT, deltamethrin, dichlorvos (DDVP), difenoconazole, edifenphos, endosulfan, ethion, fenopropathrin, fenvalerate, malathion, methamidophos, methidathion, parathion-methyl, permethrin, pirimiphos-methyl, profenophos and pyridaben. High residues were found in tea samples extracted directly with ethyl acetate when the pesticides were analyzed by the proposed method. However, after the pesticides were analyzed by a method using a hot water infusion, the residue level was decreased to less than detection limits (data not shown). Four samples $(0.26 \%)$ were in violation: chlorpyrifos in welsh onion, okra and persimmon, and fenvalerate in frozen pimiento. No detectable residues were found in 1328 (87.6\%) of the samples.

In conclusion, 128 pesticides were newly validated by the proposed multiresidue screening vegetables-fruits and cereals-seeds techniques. The vali- dation study provides a judgment that a total of 200 pesticides including 72 previously validated by the proposed methods are available to the pesticidemonitoring programme in imported foods at the Quarantine Station in Japan.

Acknowledgements The authors are grateful to Dr. Kumiko Sasaki, The National Institute of Health Sciences in Japan for her useful advice on the review of the manuscript.

\section{REFERENCES}

1) Antonious, G. F. (2004) Residues and half-lives of pyrethrins on field grown pepper and tomato. $J$. Environ. Sci. Health B, 39, 491-503.

2) Smith, L. A. and Thomson, S. J. (2003) United States Department of Agriculture-Agricultural Research Service: Research in application technology for pest management. Pest Manag. Sci., 59, 699-707.

3) Pereira, J. L., Silva, A. A., Picanco, M. C., Barros, E. C. and Jakelaitis, A. (2005) Effects of herbicide and insecticide interaction on soil entomofauna under maize crop. J. Environ. Sci. Health B, 40, 4554.

4) Elzen, G. W. and Hardee, D. D. (2003) United States Department of Agriculture-Agricultural Research Service: Research on managing insect resistance to insecticides. Pest Manag. Sci., 59, 770-776.

5) McQuilken, M. P. and Hopkins, K. E. (2004) Biology and integrated control of pestalotiopsis on container-grown ericaceous crops. Pest Manag. Sci., 60, $135-142$. 
6) Guan, T. T., Blank, G. and Holley, R. A. (2005) Survival of pathogenic bacteria in pesticide solutions and on treated tomato plants. J. Food Prot., 68, 296304.

7) Anderson, L. W. (2003) A review of aquatic weed biology and management research conducted by the United States Department of Agriculture-Agricultural Research Service. Pest Manag. Sci., 59, 801813.

8) Owen, M. D. and Zelaya, I. A. (2005) Herbicideresistant crops and weed resistance to herbicides. Pest Manag. Sci., 61, 301-311.

9) Giannakou, I. O. and Karpouzas, D. G. (2003) Evaluation of chemical and integrated strategies as alternatives to methyl bromide for the control of rootknot nematodes in Greece. Pest Manag. Sci., 59, 883-892.

10) Cowgill, S. E., Wright, C. and Atkinson, H. J. (2002) Transgenic potatoes with enhanced leaves of nematode resistance do not have altered susceptibility to nontarget aphids. Mol. Ecol., 11, 821-827.

11) Brackenbury, T. D. and Appleton, C. C. (1997) A comprehensive evaluation of Agave attenuate, a candidate plant molluscicide in South Africa. Acta Trop., 68, 201-213.

12) Tsumura, Y., Ishimitsu, S., Otaki, K., Utsumi, H., Matsumoto, N., Daba, M., Tsuchiya, T., Ukyo, M. and Tonogai, Y. (2003) An experimental proficiency test for ability to screen 104 residual pesticides in agricultural products. J. Food Hyg. Soc. Jpn., 44, 234-245 (in Japanese).

13) Van Eeden, C. M., Liebenberg, W., du Preez, J. L. and de Villiers, M. M. (2004) Solvent and surfactant enhanced solubilization, stabilization, and degradation of amitraz. J. Environ. Sci. Health B, 39, 33-51.

14) Jimenez, J. J., Nozal, M. J., Bernal, J. L., Santos, M. and Mayorga, A. L. (2002) Factors affecting the extraction, hydrolysis and derivatization steps for the quantitation of total residues of amitraz in honey by gas chromatography with electron capture detection. Anal. Bioanal. Chem., 374, 300-304.

15) Tonogai, Y., Tsumura, Y., Nakamura, Y., Matsuki, H. and Ito, Y. (1992) Studies on simultaneous determination of 12 kinds of fungicides containing unstable captan, captafol etc. in preparation of test solution. Jpn. J. Toxicol. Environ. Health, 38, 270281 (in Japanese).

16) Nemoto, S., Shimizu, R., Kikawa, H., Sasaki, K. and Saito, Y. (1995) Analytical method of Phoxim in agricultural products by GC-FPD and GC/MS (SIM). J. Food Hyg. Soc. Jpn., 36, 233-243 (in Japanese).

17) Tonogai, Y., Nakamura, Y., Tsumura, Y., Shibata, T., Kimura, M., Oota, M., Hirahara, Y., Miyata, M., Narita, M., Sekiguchi, Y., Itoyama, T., Koiguchi, S., Hasegawa, M., Miyoshi, T., Kamakura, K., Maeda, K. and Yamana, T. (1995) Effect of a component from foods on the determination of benfuracarb and carbosulfan by gas chromatography. J. Food Hyg. Soc. Jpn., 36, 506-515 (in Japanese).

18) Sphon, J. A. (1978) Use of mass spectrometry for confirmation of animal drug residues. J. Assoc. Off. Anal. Chem., 961, 1247-1252. 